\title{
Evaluating Interface Characteristics for Shared Lighting Systems in the Office Environment
}

\author{
Thomas van de Werff Karin Niemantsverdriet Harm van Essen Berry Eggen \\ Department of Industrial Design \\ Eindhoven University of Technology \\ \{t.c.f.v.d.werff; k.niemantsverdriet; h.a.v.essen; j.h.eggen\}@tue.nl
}

\begin{abstract}
IoT developments make shared systems, such as lighting systems, increasingly connected. From an interaction perspective, this offers opportunities for personal control. Especially for lighting, the benefits of personal control have been underlined by research. However, how to design interfaces that realise these potential benefits is much less investigated. This paper presents a long-term qualitative study in which three interfaces for a shared lighting system are evaluated by 17 people working in an open plan office. The interfaces are designed to vary on a number of characteristics, including the distribution over space, interaction modality, and sequence of interaction. Based on the results, we provide new insights in the impact of interface characteristics on lighting use and experience. We find, i.a., that having an interface on a personal multipurpose device or on a central interface solely dedicated to lighting, influences whether people make individual or more collective lighting adjustments and decisions.
\end{abstract}

\section{Author Keywords}

Interface Characteristics; Shared Interaction; Connected lighting systems; Personal control; Internet of Things (IoT); Work environment; Open plan office; Interaction Design.

\section{ACM Classification Keywords}

H.5.2. Information interfaces and presentation (e.g., HCI): User Interfaces - Interaction styles / User-centred design

\section{INTRODUCTION}

The Internet of Things (IoT) paradigm has been rapidly gaining ground in our daily lives, resulting in systems becoming increasingly connected. This brings many advantages for personalization of an environment, service provision and system management. One of the most evident recent examples are connected lighting systems for office environments. This development offers potential benefits,

\footnotetext{
Permission to make digital or hard copies of part or all of this work for personal or classroom use is granted without fee provided that copies are not made or distributed for profit or commercial advantage and that copies bear this notice and the full citation on the first page. Copyrights for thirdparty components of this work must be honored. For all other uses, contact the Owner/Author.

Copyright is held by the owner/author(s).

DIS 2017, June 10-14, 2017, Edinburgh, United Kingdom

ACM 978-1-4503-4922-2/17/06.

http://dx.doi.org/10.1145/3064663.3064749
}

including cost and energy savings in both installation and operation; adaptation of other building systems for (remote) building management, facility services, security provision and data management; and - most interestingly - personal control for office workers over their environment [44]. The benefits of personal control over lighting have been studied widely, especially in the office environment. Personal control can decrease energy consumption $[6,7,46]$, increase work comfort [38] and appraisal of the work environment [43], and improve job satisfaction [42]. These are clearly desirable benefits, especially with the increasing number of open plan offices where people share space and desks with co-workers [23]. However, how to design lighting interfaces that realise these potential benefits is a much less explored area.

Traditionally people interact with office lighting through switches, turning light on or off. However, developments in LED technology give more detailed control possibilities, thus requiring a new interaction paradigm [25,32]. Most commercially available connected lighting systems currently present mobile phone applications to interact with the light, both in the home environment $[5,13,33,35]$ and in the office environment $[10,34,36]$. The reasons for doing so are understandable: mobile phones are a widely available and highly advanced platform, applications are relatively cheap and endlessly scalable, and people are getting used to this means of interaction. Nevertheless, their suitability for lighting interaction has also been questioned. Taking the phone out of your pocket, locating and activating the app takes effort and time [4,32]. Also, usually not everyone has access to the application or network connection; e.g., [29]). Moreover, mobile phones offer highly personalized interaction styles - often based on personal preferences and presets - while the resulting light setting is likely to influence multiple people that share the lighting output $[29,30]$. In research, an array of alternative lighting interfaces has been proposed (e.g., $[1,4,9,18,24,29,40])$ but they have rarely been evaluated in use. Hence, the impact that interface characteristics have on the user experience of these systems is not fully known.

The study that we present in this paper has two main aims: to investigate (1) how different user interface characteristics influence use and experience with lighting systems and (2) how a representation of social information in the interfaces can help people to take each other into account while 
interacting with the lighting system. We expect that insights from this study guide the design of interfaces for shared lighting systems. Since both aims require a separate detailed analysis, this work focuses on the first aim by investigating the influence of interface characteristics on use. To address this aim, we take an explorative researchthrough-design approach [47]. We design three interfaces that offer the same control over the light but that vary in distribution over space, being single- or multi-purpose, and ownership; interaction modality; sequence of interaction; representation of the light in a preview; and social information. The resulting three interfaces - the Floorplan, Pointer, and Canvas - are evaluated for approximately a month each with 17 participants working in an open plan office. Based on the results, we provide new insights in the impact of interface characteristics on lighting use, and we argue to carefully consider these characteristics in the design process.

\section{RELATED WORK}

Personal control (beyond the traditional light switches) in the professional work environment is a relatively new development but several systems have recently become commercially available. Philips connected lighting [36] and Osram Lightify Pro [34] offer office workers a mobile phone application to control the light intensity and room temperature. Connected lighting systems are more common for the home environment (e.g., [5,13,33,35]). Most of these systems offer interaction through mobile phone, tablet, and smart watch applications. In some cases, dedicated switches or remotes are added [22,37]; or connection to home automation systems $[2,17,48]$ can be made to open up a range of alternative interaction styles, including connection to sensors and speech.

In research, a large range of lighting interfaces has been presented for the home environment. Tangible lights [40] present in-air gestural controls over lighting that is inspired upon giving a light beam physical qualities. The Reality Editor [18] maps graphical user interface elements on top of a real-time video capture of the lamp through augmented reality techniques. Bakker and Niemantsverdriet [4] present four tangible and tangible-gestural interfaces for domestic lighting systems that can potentially be interacted with in the periphery of attention - i.e., without attending to explicitly [3]. The case for peripheral lighting interaction had been made before by Aliakseyeu et al. [1], since lighting is usually not a goal in itself but more "a means to an end" ([1], p. 219). To our knowledge, there are much less interface proposals in research for personal lighting control in office environments. Magielse and Offermans [27] present a range of interfaces for a breakout area within the office, consisting of a preset-cube for atmospheric light, a touchpad to control direct light, and multiple screen-based apps for more detailed lighting control [27]; described in more detail in [1]. The Bolb [26] is a portable handheld device for workers in shared office spaces that lets people create a personal 'light bubble' that follows them around.
While all interfaces present interesting possibilities, many are one-off designs and many are not evaluated.

A more systematic evaluation of interface characteristics is presented by Brumitt \& Cadiz [9]. They compare a number of different lighting interactions: plain text computer displays, graphical user interfaces, voice control, and gestural interactions; all worked out into Wizard of $\mathrm{Oz}$ interfaces [11]. The evaluation sessions were done in a smart home lab. Less related to specific interface proposals, ethnographically inspired probes have aimed at finding overarching interaction requirements for lighting interaction at home. Lucero et al. [24] studied interactive lighting opportunities in bathrooms and concluded that opportunities are limited by people's concerns about the potential complexity of the interaction. Offermans et al. [32] investigated people's current everyday interaction with lighting systems. They found that lighting needs are mainly latent and highly dependent on context. Overall, the ethnographic studies present interesting insights but they are not based on actual interaction designs and therefore more difficult to implement directly.

\section{INTERFACE CHARACTERISTICS AND DESIGNS}

In this work, we aim to systematically evaluate how variations of five interface characteristics influence the use of a shared lighting system in an office environment. In this section, we first explain the characteristics and their variations when implemented in interface designs. We then present three lighting interfaces that each embody a different combination of such variations (see Table 1).

\begin{tabular}{c|c|c|c}
$\begin{array}{c}\text { Ownership } \\
\text { and } \\
\text { distribution }\end{array}$ & $\begin{array}{c}\text { On a personal } \\
\text { device }\end{array}$ & $\begin{array}{c}\text { Distributed } \\
\text { per table } \\
\text { group }\end{array}$ & $\begin{array}{c}\text { One shared, } \\
\text { central } \\
\text { interface }\end{array}$ \\
\hline Purpose & Multi-purpose & Dedicated & Dedicated \\
\hline $\begin{array}{c}\text { Interaction } \\
\text { modality }\end{array}$ & $\begin{array}{c}\text { Graphical } \\
\text { user interface }\end{array}$ & $\begin{array}{c}\text { Tangible- } \\
\text { gestural } \\
\text { interface }\end{array}$ & $\begin{array}{c}\text { Tangible } \\
\text { interface }\end{array}$ \\
\hline $\begin{array}{c}\text { Sequence of } \\
\text { interaction }\end{array}$ & $\begin{array}{c}\text { Starting from } \\
\text { the location }\end{array}$ & $\begin{array}{c}\text { Starting } \\
\text { from light } \\
\text { parameters }\end{array}$ & $\begin{array}{c}\text { Combining } \\
\text { parameters } \\
\text { and location }\end{array}$ \\
\hline $\begin{array}{c}\text { Represen- } \\
\text { tation of light }\end{array}$ & $\begin{array}{c}\text { Screen-based } \\
\text { graphics }\end{array}$ & $\begin{array}{c}\text { Light-based } \\
\text { (LED) }\end{array}$ & $\begin{array}{c}\text { Printed } \\
\text { graphics }\end{array}$ \\
\hline $\begin{array}{c}\text { Social } \\
\text { information }\end{array}$ & Intention & Identity & Preference \\
\hline
\end{tabular}

Table 1. An overview of the interface characteristics represented in the three interfaces.

\section{Interface Characteristics}

1. Ownership over the interface, Distribution over space, and Single-/Multi-purpose devices.

Choosing for a mobile device for interaction with a lighting system defines characteristics such as distribution, ownership, and purpose. Since mobile devices (e.g., laptops, smart phones, smart watches and tablets) are 
generally personal, they are unlikely to be shared with others, resulting in a fine-grained distribution of interfaces over space. Also, such a device is not dedicated to lighting control only, as it is used for more purposes (such as work, communication, or entertainment). Characteristics as the location and the number of people that share an interface need to be carefully considered when designing a dedicated lighting interface. For example, Offermans et al. [32], found that the location of the interface greatly influences effort, and thus the willingness to interact - people often do not bother to move in order to adjust the light. In this sense, personal devices present an interesting paradox in availability of lighting control. Personal devices are mobile and at hand but finding and opening an application still requires effort and time, which is expected to lower availability $[1,32]$.

In this study, we aim to make three distinct combinations of distribution (at hand, at the table, centrally in the space), ownership (personal, shared with a small group, and shared with everyone), and purpose (single- or multi-purpose).

\section{Interaction Modality}

Applications are screen-based graphical user interface (e.g., [13,34-36]), but other interaction modalities can also be considered. Two examples are tangible $[19,41]$ and gesture-based interaction styles [21], which "rely on embodied interaction, tangible manipulation, physical representation of data and embedding in real space" ([19], p.437). Tangible interaction is known to have potential advantages over screen-based interaction styles. For example, tangibility can lead to increased directness and better understanding of mapping between input and output, since the state of the system can generally be directly read from the manipulated object [20,41]. Moreover, tangible interaction appeals to people's perceptual-motor skills, rather than focusing only on cognitive skills [12,20]. Because of this last quality, tangible and embodied interactions are seen as a valuable approach to design interaction in the periphery of attention [3,4]. Tangible gesture interfaces have good embedding in space, which can be useful when addressing individual lamps. Advantages of screen-based approaches are the ability for higher complexity in feedback [20]. Also, interaction elements in digital interfaces are more scalable (e.g. the number of individually addressable lamps). Lastly, people are accustomed to this type of interaction nowadays, as screen-based interfaces are omnipresent.

In this study, we compare three types of modalities: a tangible, a tangible-gestural, and a screen-based graphical user interface, to investigate how the benefits of the different modalities apply to lighting interaction.

\section{Sequence of Interaction}

One should carefully consider how light parameters are presented to the user of the interface, as people are generally unfamiliar with detailed lighting control $[24,25,32]$. Even without variations in the number of controllable parameters, different layers of control can be offered. Offermans ([31], p.134) describes a model consisting of three levels of light parameters: context, lighting, and lamp parameters. For example, ambient presets (as recommended by [24]) regard context parameters, where lamp parameters refer to technical settings of individual lamps (intensity, hue, saturation). However, which of these control parameters (light parameters, location in space, or lamps in a group) is a starting point in the sequence of the interaction, and how their relations are visualized still make an important design consideration. For example, the Philips Hue application [35] the sequence of interaction starts with the location of lamp groups: the main menu shows the areas to which lighting settings can be applied. But on a deeper interaction layer, the lamp parameters are central: lamps can be placed on a field of hue and saturation.

In this study, we make use of Offermans' lighting layer in the three interfaces, where we let people control parameters such as colour temperature and intensity of groups of lamps pertaining to a certain area in the space. We aim to compare three different sequences of interaction, presenting different mappings of lighting, lamps and space. In the first interface, the location for the adjustment is first selected after which light settings can be applied to that location. In the second interface, first a light setting is made that can then be applied to specific lamps. And in the third interface, lamps can be placed in a field of light settings.

\section{Representation of the Light in the Interface (Preview)}

In general, people are not acquainted with technical light parameters [25]. Control over intensity is more or less known because of conventional dimmer controls, but parameters like colour temperature, hue, or saturation are relatively new. Therefore, it might be challenging to find the right light setting instantly. An interface can support people in estimating the effect of an adjustment on the context, before the light setting is applied [45]. But the appropriateness of the medium in which this representation is expressed is unknown. The prediction accuracy of lighting representations of photographs and renderings have been studied (e.g., [14,39]), but such representations are much more detailed than the abstracted representation we envision.

In this study, we compare different media for previewing the light adjustment: digital graphics (on a backlit OLED or LCD screen, as is the case in most applications), an LED light source, and printed graphics that do not involve light.

\section{Social Information}

The final characteristic for the interfaces is the social information about other people in the space that is represented in the interface. Making socially salient information visible in order to make people aware of each other's needs, wishes, and motivations, resulting in accountability over an interaction; is known as social translucence [16]. We have previously emphasized the 

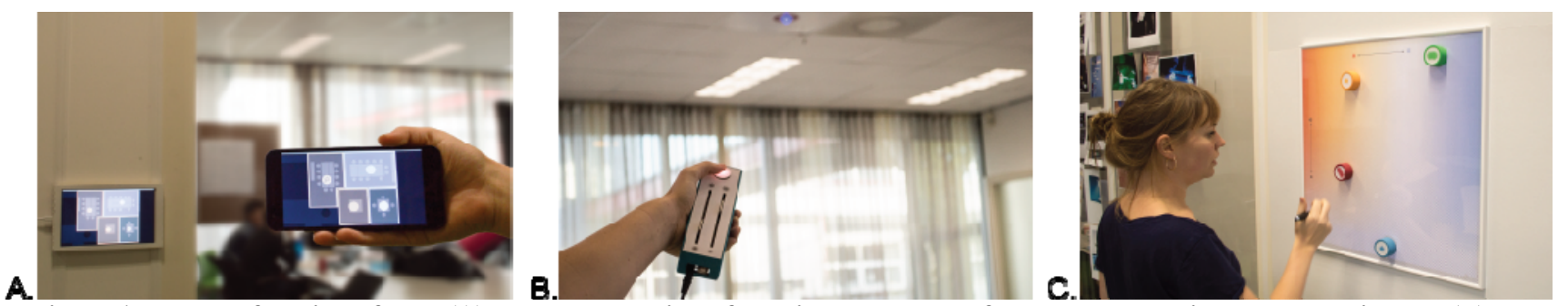

Figure 1. Photos of the interfaces: (A) the Floorplan interface displays a map of the space were light can be adjusted; (B) the Pointer as remote control for the light; and (C) the Canvas interface with tokens that can be moved around to change the light.

importance of social translucence in interfaces for shared systems [29,30], and we have presented first ideas on how this information could be presented in lighting interfaces $[29,45]$. But the relevance of the type of social information is yet unknown. Therefore, we vary three different types of social information in the interfaces: (1) intention with the new light setting; (2) identity of the person that made the last lighting adjustment; and (3) preference of the people in the space. As mentioned in the introduction, the evaluation of this characteristic is outside of the scope of this paper.

\section{Interface Designs}

We iteratively designed three interfaces ${ }^{*}$ that combine variations of the characteristics: The Floorplan, Pointer, and Canvas interface (figure 1). These interfaces vary on their designed characteristics, not on the level of control they offer. Therefore, all three interfaces can be used to control intensity $(0$ to $3800 \mathrm{~lm}$ ) and colour temperature (1700$8000 \mathrm{~K}$ ) of four clusters of lamps. While the interfaces are one-off prototypes, they are robust and fully functional.

\section{The Floorplan interface}

The Floorplan interface (figure 1A) is a graphical user interface that can be installed on a personal device and is available on a tablet located centrally in the space (figure 5). Central in the interface is a map of the space, indicating the layout of the tables and the four clusters of lamps. To start the interaction, users can select one or more clusters by drawing on the map (figure 2A). Next, the user can control sliders to adjust intensity and colour temperature within the selected area (figure 2B). The circle in the centre of the controls gives a representation of the new light setting (the preview). Only by pressing the preview circle, the setting is applied to the space. After setting the light, the user can select an activity to indicate their intention with that light setting to others (figure 2C). The icon and the light setting itself remain visible on the interface for other people in the space. Active instances of the application are synced without noticeable delay, and thus always display the current status of the luminaires (figure 2D).

The application is made with Processing [49] and exported as an application for Windows, Apple OS, and Android devices. The application communicates with the local server PC over Wi-Fi. The server uses broadcast messages to make sure all Floorplan apps are in sync with each other.

\footnotetext{
${ }^{*}$ Video of the interfaces: vimeo.com/twerff/sharedlighting
}

\section{Pointer Interface}

The Pointer interface (figure 1B) is a tangible-gesture interface, which can be used as a remote control for the light. A remote control, or sender, is positioned on each table area in the space. Above each table area, a receiver is installed in the ceiling, which is visible from every position in the space. Every table area is given a colour. The senders and corresponding receivers have a colour corresponding with their respective table. The sender has two sliders that can be used to adjust intensity and colour temperature (figure 3A). A button with an integrated RGB LED shows a preview of the new light setting. By pointing the sender to an area and pressing the button, the light setting is applied to the lamps in that area (figure 3B). The indication light in the targeted receiver briefly flickers to confirm that it received a message, and takes on the colour of the sender that made the adjustment. This indication light shows the colour until it is targeted by a different sender. In this way, people can always see who set the current light setting. By pointing at other areas and pressing the button again, the setting can be applied to multiple areas (figure 3C).

When the button is pressed on the sender, the ID of the sender is transmitted to the targeted receiver using a coded infrared (IR) signal. The sender comprises an IR LED (TSAL6100), 2 analogue linear slider potentiometers, a Lithne board [28], and a button with an integrated RGB LED, in a laser cut MDF casing with acrylic cover. The receiver comprises an IR receiver (TSOP32438), a Lithne board [28], and an RGB LED with diffuser, integrated in a cardboard ceiling tile.
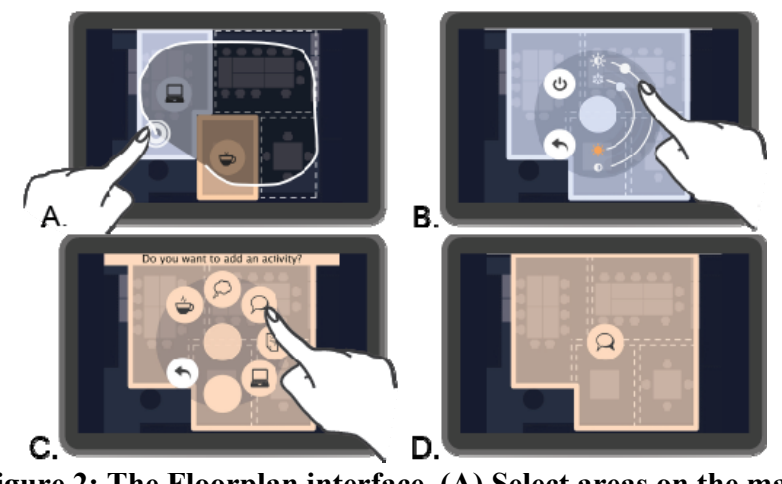

Figure 2: The Floorplan interface. (A) Select areas on the map. (B) Adjust intensity and colour temperature and press the preview to apply. (C) Select an activity to show intention (optional). (D) The interface shows the current status. 


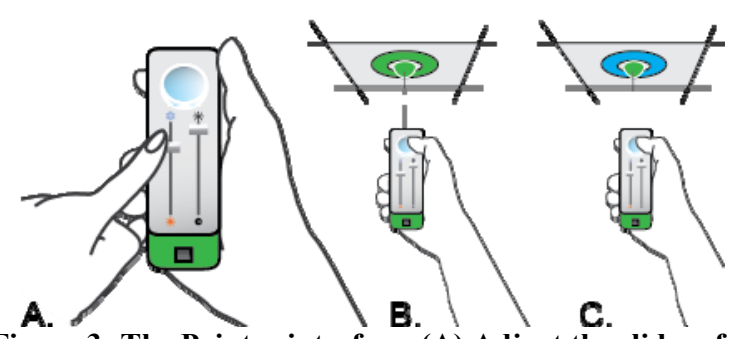

Figure 3: The Pointer interface. (A) Adjust the sliders for intensity and colour temperature. (B) Point to an area and press the button. The indicator shows the sender's colour. (C)

Point and click to other areas to apply the same setting.

\section{Canvas Interface}

The Canvas interface (figure 1C) is a tangible user interface located in a central location in the space. The four areas are represented by tokens with a colour and an icon in the shape of the corresponding table group. Tokens can be moved around on a gradient map with two axes: the colour temperature on the horizontal axis, and intensity on the vertical axis. The gradients are printed on the canvas to provide a preview of the light adjustment. To adjust the light, a token can be repositioned on the map. The new values for colour temperature and intensity of the token's position are applied directly to the lamps in the cluster corresponding with that token (figure 4A). By drawing a line around one or more tokens with the boundary stylus, the user can show to others how much change to the light is accepted (figure 4B). Boundaries thus show light preferences of people in the room. Boundaries can be removed by briefly tapping them with the stylus or by moving the token(s) out of the boundary.

The Canvas interface comprises passive physical tokens, made of MDF and magnets, on a photo frame with a semitranslucent print between two layers of acrylic. Markers on the back of the frame are connected by magnets to the tokens on the front of the frame. Sliding a token over the Canvas makes the magnetic marker follow. The position of the markers is detected by a webcam connected to a PC running a Processing sketch [49]. The boundary stylus is a laser pointer that is tracked through pixel colour detection. The boundaries are projected again from the back onto the Canvas. The interface is placed in an open doorframe to hide the projector, webcam and computer from sight.

A.
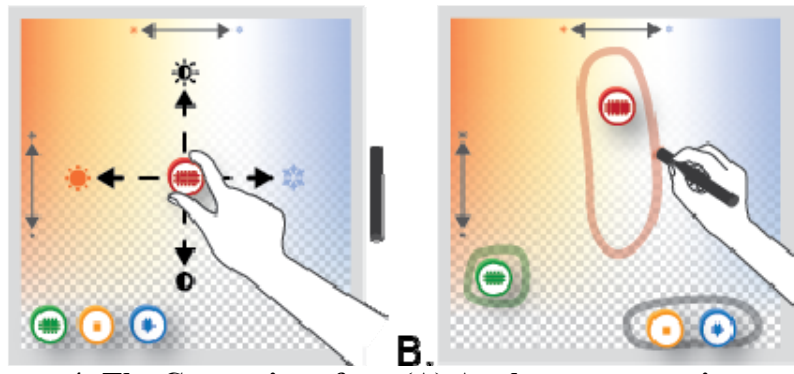

Figure 4: The Canvas interface. (A) A token, representing an area, can be moved freely over the canvas: vertical to adjust intensity; horizontal for colour temperature. (B) A boundary can be drawn with the stylus, indicating preference to others.

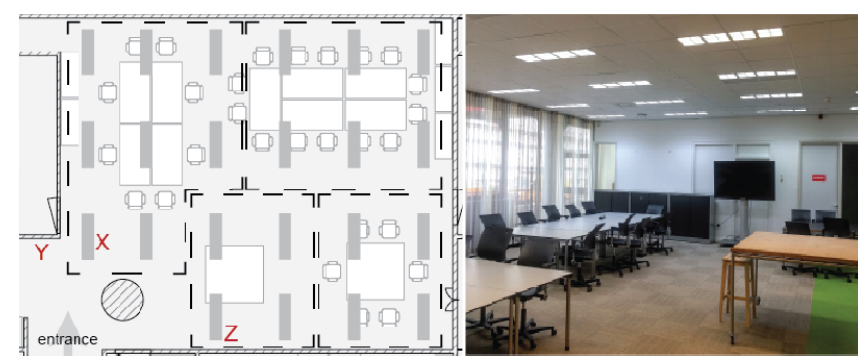

Figure 5. A map of the office with the lamps (grey) in 4 areas (dashed lines), the tables, the position of the central tablet (Y) and the Canvas $(\mathrm{Z})$. The photo is taken from position $\mathrm{X}$.

\section{EVALUATION SETUP}

To make sure that the insights on how interface characteristics affect daily use represent real life interaction, it is important to evaluate the interfaces longitudinally and in a real work environment. Since connected lighting systems are not widely available yet, we equipped an open plan office at the Eindhoven University of Technology (figure 5) with a connected lighting system. This system is the only light source, apart from little daylight from northfacing windows. The office measures $88 \mathrm{~m}^{2}$ and provides space for up to 28 people. It is occupied daily by students from the department of Industrial Design, and is used for a variety of activities. The space can be easily observed, allowing for a quick response to (technical) issues.

The connected lighting system is a prototype system developed by the Enlight consortium [15] and comprises 22 custom LED luminaires. Each luminaire has integrated logic and sensing (motion, light level, and temperature) and its light output can vary in intensity $(0-3800 \mathrm{~lm})$ and colour temperature $(1700 \mathrm{~K}-8000 \mathrm{~K})$. The luminaires can be addressed and controlled individually by the server, using the ZigBee protocol for wireless communication. As said, for this study control was restricted to four groups of luminaires. The groups are predefined to match the four table groups in the space (see figure 1). In any given moment, the groups can differ in light setting, but individual luminaires cannot be addressed and luminaires cannot change group. A server PC runs a Processing sketch [49] that processes incoming messages from the lighting interfaces to usable commands for the luminaires. The Floorplan and Canvas interfaces communicate with the server through UDP messages over Wi-Fi. The senders and receivers of the Pointer interface communicates with the server over a second ZigBee network.

\section{Method}

The three interfaces were deployed in the living lab for 4 to 5 weeks each. The 14-week study was conducted during the university semester from September to December as these are dark months in the Netherlands, ensuring a need for artificial lighting in the space. Participants were recruited during an introductory presentation for bachelor and master students involved in a project on lighting interaction at the faculty of industrial design. Participation was voluntarily and not a part of the educational process. The performing 
researchers had no formal role in the educational project and assessment. A total of 18 participants signed up for the study. One person stopped participation in the first 4 weeks and was therefore not included in the results. The fact that all participants (age 20-30, $\mathrm{F}=5$ ) were involved in a project about lighting interaction helped in sensitizing them for study. The office has flexible workplaces and is open to all students of the department. Therefore, the interfaces were used not only participants, but by others (visitors) as well. To facilitate use, we spread manuals for the interfaces over the space. We cannot distinguish participant's interactions from the interactions of visitors in the gathered log-data.

Figure 6 shows a schematic setup of the study. The Floorplan was implemented first as the screen-based application was expected to correspond with people's experience and expectations of interacting with a connected system. The Canvas was implemented last as the tokens representing areas were expected to be most complex to learn. The Pointer as second, introduced the colour coding.

Data was collected in three ways. First, every incoming message from the interfaces was logged, including time and date, the sender of the message (interface or participant ID), and the adjusted light parameters (the adjusted area, colour temperature, and intensity). Second, a weekly data sampling survey was sent out to each participant via email (the circles in figure 6). The survey requested motivation and a description of context for a specific moment of interaction. In this way, the survey was a weekly reminder of the ongoing study and triggered reflection upon peoples' experiences. Third, three semi-structured interviews were conducted with each participant individually during the last week of each interface use-period (blue lines in figure 6).

The interviews included questions about general use of lighting and space, specific interactions with the interface (referring back to the results from the data sampling surveys), and evaluation of the interfaces. To fuel the conversation on evaluation of the interfaces, we presented the interviewees with a five-point Likert scale survey on specific features (e.g., 'How enjoyable was the interface?', and 'How much effort did interaction take?'). We concluded the third and final interview with an extra 20minute part where participants compared their experiences with the three interfaces by positioning magnetic images of features of each interface (e.g., 'quality of the preview', 'precision of control', 'invitation to interact') on 5-point scales (figure 7). All interviews were audio-recorded with permission. 15 out of 17 participants participated in all interviews, one person missed the second interview and one missed the concluding interview.

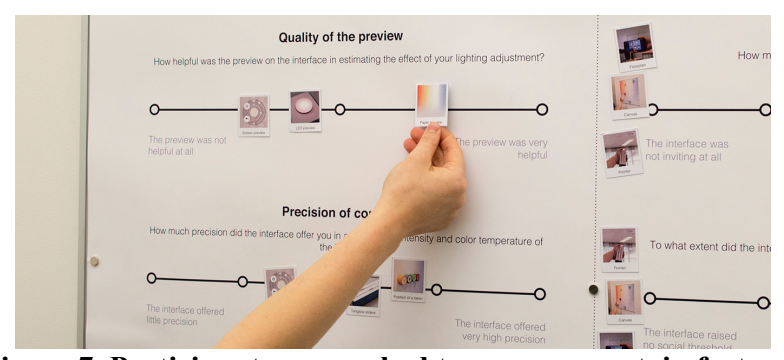

Figure 7. Participants were asked to compare certain features of the three interfaces on a number of 5-point scales.

The setup was piloted with seven $\mathrm{PhD}$ students that were asked to work in the living lab for one week. During this week, they used the Floorplan interface to control the light and received three data sampling surveys. At the end of the week, five participants received a closing questionnaire and two were interviewed. Next to minor adjustments in the interface and the resolving of stability issues, the introduction and explanation of the interface was refined and the concluding questionnaire was replaced by a semistructured interview with the Likert scales.

\section{Analysis}

Quantitative data was used to analyse and demonstrate general use during the study of the space, lighting, and interfaces. This includes the log data from the interfaces and self-reported number of interactions and presence in the space from the three interviews. Log data from the first two days of each period was excluded as technical fine-tuning happened during those days. Since one interaction can comprise of multiple separately logged messages (because of, for example, fine-tuning), the separate messages were clustered where applicable.

We based most of our results presented in this paper on the analysis of qualitative data. This data includes the recorded audio from the concluding section of the final interview, since this part focused most directly on the comparison of the characteristics of the interfaces. The remaining qualitative data from the weekly surveys and the other interviews is to be used in a later, more extensive analysis, focusing on the social information and multi-user interaction. The results from the Likert scale questionnaires in the interviews were used to make subjective evaluation of the interfaces comparable amongst participants.

The audio recordings were transcribed verbatim, and then used in a thematic analysis [8]. First, quotes from the 16 interview transcriptions were selected, resulting in 173 unique quotes (between 5 and 21 quotes per interview). The quotes were collaboratively coded and clustered using qualitative analysis software MaxQDA [50], resulting in the following themes: general use ( 29 quotes), interface

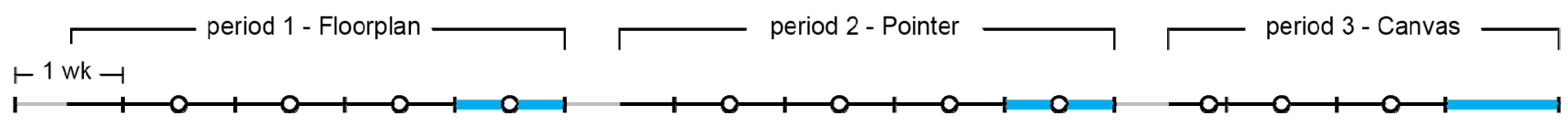

Figure 6. An overview of the study, including the log data collection period of three interfaces (black line), the data sampling questionnaires (circles), and the interview weeks (blue line) 
evaluations (43 quotes), and the four interface characteristics (74 quotes on distribution, dedication, and ownership, 17 for interaction modality, 54 for sequence of use, and 32 for representation of light). Within each theme, we iteratively reviewed the quotes to identify recurring patterns, especially looking for effects of the characteristics on user experience and use of the lighting system. The identified effects within characteristics were compared amongst each other, resulting in five distinct effects: Sharedness of an interaction, Effort, Reminder to interact, Engagement, and feeling of Control. Figure 10 displays a visual overview of the relation between characteristics and effects, which is further elaborated in the next section.

\section{RESULTS}

In this section, we first describe how the space, the lighting, and the interfaces were used during the period of the study. Second, a brief evaluation of each interface by the study participants is described. Third, the effects of the interface characteristics on user experience are described per characteristic. The findings are illustrated with example quotes and quantitative results.

\section{General use}

During the test period, participants interacted 290 times with the Floorplan, 413 times with the Pointer and 351 times with the Canvas (see figure 8 for an overview of the number of interactions per interface per day). There was a noticeable decrease in usage over time for each interface, as it became more familiar to the participants. All interfaces were used at least once each day.

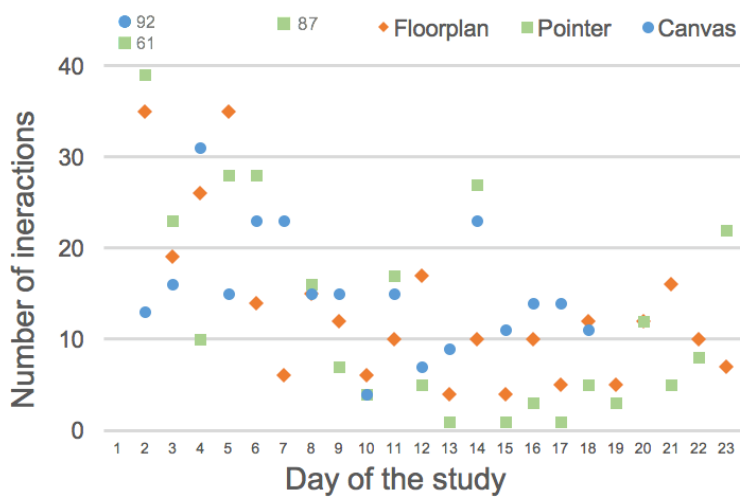

Figure 8: Number of interactions per day, per interface.

Since the Floorplan interface can identify each user through his/her IP address, we know the number of interactions per person for this interface. There were between 1 and 25 interactions per person with the Floorplan during the fiveweek period (see figure 9). This difference can be explained by interpersonal differences on how important good lighting is, as well as by the variance in presence. Over the whole study period, $67 \%$ of the participants was in the space at least a couple of times per week. On average, when people were present they interacted with the interfaces at least once. This seems to be the same for all three interfaces, judging from self-reported presence and use of the Canvas and Pointer.

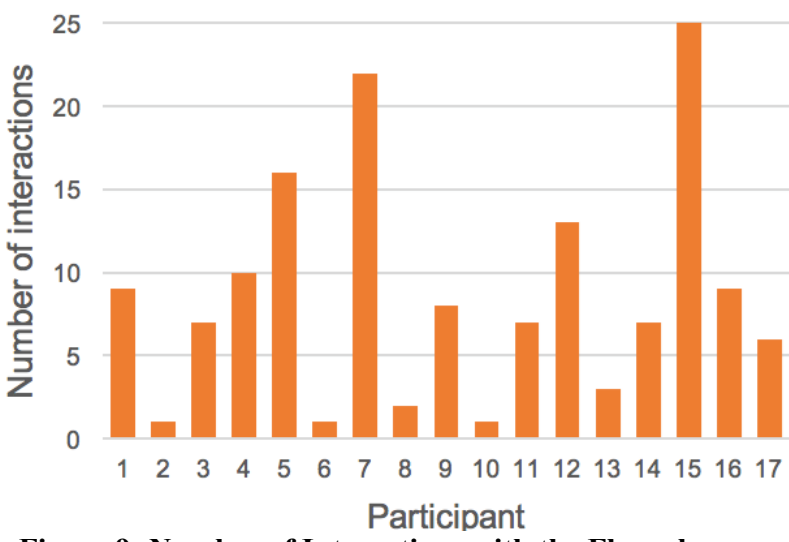

Figure 9: Number of Interactions with the Floorplan per participant. An additional 143 interactions were done by nonparticipating visitors.

In most interactions, people adjusted the lighting in one area $(54 \%)$, or in all four areas in the space at once $(24 \%)$. In the interviews, participants explained that most of the time they only adjusted the lighting in the area that they were located in. "I would only, mainly, adjust light above the table where we sat. I didn't really think about changing it above the other tables because there were people there and I didn't know their preferences." (P14). When no other people were present, they would consider making adjustments to the whole space: "Suppose I was sitting in an empty space in the dark. I would not change only my own lamps; I would change everything. Otherwise it feels weird." (P11). We noticed a slight difference between interfaces: with the Floorplan, $41 \%$ of the interactions adjusted one area, while this is $66 \%$ and $52 \%$ respectively for the Pointer and Canvas. With the Floorplan, in $40 \%$ of the interactions the whole space was adjusted; much more than with the Pointer (12\%) and Canvas (25\%).

\section{General Evaluation of the Interfaces}

The Floorplan was understandable: it matched people's experience of interacting through applications. "[The Floorplan] is not hard to interact with [...] Because you use applications and stuff all the time, so it's not hard. It is just less fun." (P6). People generally valued the possibility of controlling light on a personal device: 12 participants installed the Floorplan on their laptop, of which 10 also used the application on their laptop. And 9 out of 10 participants that installed the application on their phone, used the application on their phone. $59 \%$ of the participants $(10 / 17)$ used the application on the central tablet. The interface had some stability issues, which resulted in a decreased feeling of control. "Since the system was down and the response was sometimes a bit late, the feeling of control was lost sometimes." (P14). For some participants, the area selection through drawing on the floorplan gave the illusion of control over individual lamps instead of the predefined clusters. Also, most participants found the sliders too small on their phone application. 
Most of the participants enjoyed the Pointer interface, especially because of its physical presence and availability. Directing and clicking - 'shooting' - was enjoyable and playful. "You could shoot with it, which was fun. [...] Personally, I liked the Pointer the best, because it was there in the middle of the table: anyone could take it easily. It was clear what you were adjusting with that preview light, and it was not overly complicated." (P17). Participants mentioned that it could be difficult to precisely target the receiver, leading to some trial and error: "Sometimes [...] I had to press the button a number of times." (P1).

The Canvas was well received with all participants. It was described as surprising and engaging, and participants enjoyed playing with the physical tokens. The size and location of the interface made it visible from anywhere in the space. "I enjoyed the [Canvas] most. It did take a bit more time but it invites you to play, to change something. It also gave a bit more precision; or maybe not precision but it is more visual." (P4). We observed that many participants presented and explained this interface to visitors and colleagues. Most participants mentioned that walking up to the interface was effortful, especially in comparison with the Pointer that was closer by, but few refrained from interacting because of this extra effort.

\section{Interface characteristics}

The interfaces were designed to vary on several characteristics. Figure 10 provides an overview of the four characteristics (left) and the five effects on people's experience with the lighting (right) that we identified in the thematic analysis. The arrows show the relations we identified between characteristics and effects. Next, we describe these relations for each interface characteristic.

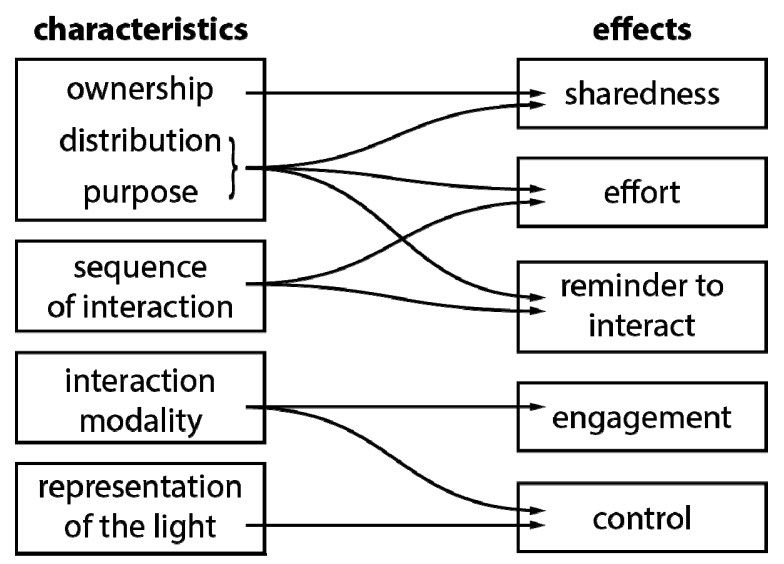

Figure 10. Relational overview of characteristic and effects.

\section{Ownership/Distribution/Purpose}

The interfaces differ in their distribution over space: The Canvas is placed in a central location, the Pointer is located per table group, and the Floorplan is both distributed (on laptops and phones) and on a central tablet. In all cases, people mentioned that if an interface is not within reach, this costs effort and raises the threshold to interact. "[The Canvas] did invite, but it had that barrier of getting up and going to it. So, it was easier to say: 'OK, it is fine as is'.,' (P12). In line with findings of Offermans et al. [32], we found that taking out a phone and starting an application also requires effort, even though the phone is usually within reach. "It is funny that I find it more difficult to get my phone than to walk across the room. I just don't like getting my phone out at all." (P6).

The three interfaces also differ in ownership: The Floorplan is personal, the Pointer is shared per table, and the Canvas is shared with all people in the room. We observed an effect on the feeling of 'sharedness' of a lighting adjustment. People felt that the adjustment was an individual or a collective decision: "I always consulted others, except with the [Floorplan]. I did say something to others, but in the end, you do it alone. And with [the Pointer and Canvas] you do it together." (P17). With the non-personal interfaces, people engaged others more in the adjustment and they wanted to be engaged when others interacted, leading to more conversations about the light. "[With the Canvas], everyone notices you walking to the interface, and thinks: 'that person is going to change the light, we need to pay attention.' With the [Pointer] the same, but it is only visible to a smaller group of people." (P13). A requirement for this collective decision making (or sharedness) is visibility of one's actions, making people accountable for them, as also stressed by [16]. Naturally, the location of the interface is important to achieve this visibility, as well as the interface being dedicated solely to lighting interaction: "If you pick up the Pointer, other people at the table notice that right away, which makes it easier to start a conversation. With the Floorplan application, other people do not see that you are interacting with the light, which makes it awkward to start a conversation about the light." (P11). A second effect of this visibility is that the interface can become a reminder to interact: "I just forgot about the [Floorplan] because I didn't see it. Even though it is in my pocket, I don't always check my phone. And even if I do, I still forget that I have an application for that." (P5).

\section{Interaction Modality}

The interfaces implement three different interaction modalities: The Floorplan is a graphical user interface, the Pointer is tangible-gestural, and the Canvas is tangible. Many participants preferred tangible interfaces to screenbased applications in general: "I'm not really fond of using an app" (P16). This might be explained by a bias in the participant group consisting of interaction design students. However, in most cases tangibility was experienced as more engaging and fun: "I think I would rather use the [Canvas] than a web application, even though I have always said that I prefer web interfaces. [...] but I like using the tokens way more." (P4). When asked how enjoyable interaction was, $75 \%$ of the participants rated the Floorplan 3 or higher (means: 3.6 ), while $75 \%$ scored a 4 or higher for the Pointer (means: 3.9) and Canvas (means: 4.3), on a scale of 1 (not enjoyable at all) to 5 (highly enjoyable). 
The physicality of controls in the tangible interfaces made it easier for participants to reach a feeling of control; both with the tokens on the Canvas and, to a lesser extent, with the sliders on the Pointer. On a scale representing precision of control, ranging from 1 (low precision) to 5 (high precision), participants rated the Canvas higher with a smaller spread in ratings (avg: 4.2, range: 2.0) than the Floorplan (avg: 3.4, range: 3.8). This finding is in line with Bakker et al. [3], who state that tangible interaction potentially requires less attention. However, it might be an effect of the digital sliders being quite small in the Floorplan.

The gestural expressivity of the Pointer was expected to increase visibility of the interaction, which can be important for the previously described sharedness. However, this was not mentioned by participants, even though the physicality of both the Canvas and the Pointer was used for this purpose: "[With the Floorplan] there is no way to communicate that this is my light. But [with the] Pointer, placing it close to me would make me feel like the owner of the area. So, someone reaching out and taking it to control the light would feel like an intrusion in my area." (P12).

\section{Sequence of Interaction}

The interfaces vary in interaction sequence. In the Floorplan, areas are selected after which a light setting is made and applied; in the Pointer, a light setting is created and then applied to an area; and in the Canvas, areas are placed on a field of light parameters, making selection of location and parameters a combined action. All three interfaces provided an understandable sequence of interaction. The overview of the map in the Floorplan was valued: "It feels nice to have the little map, saying do this and this. It's like playing age of empires." (P14), although orientation on the map could take some participants time to learn. Also, as explained earlier in this section, people mainly adjusted their own area, making the map less appropriate: "Actually, I never felt the urge to adjust more than my own area. [...] The [Floorplan] provides an easy way to do this, but I just don't need the option." (P11). The directness of pointing in the space with the Pointer was easier to learn but again, since people hardly adjusted other areas than their own, people regarded the pointing action needlessly effortful. Participants were surprisingly positive about the area-tokens on a grid of light parameters in the Canvas. The relation between tokens and table areas was expected to be difficult for people as it is a cognitive connection. Some participants mentioned this took time to learn. However, this mapping seemed to fit the general lighting use better: "Of course you need distinct token colours, it would be difficult if they become too similar. But after a few days, I knew my table was orange, and from then on you know that is the one you need to use." (P4). Overall, it seems that one sequence of interaction is not inherently better than another but that it should be carefully designed to fit context, the controllable parameters, and use of the system.
Next to the order of parameters, the interfaces also differ in the number of actions required to make an adjustment. In the Floorplan, an area is selected, then parameters are adjusted, the setting is applied, and an activity is selected (optional), leading to a multi-step sequence. The Pointer has two steps: adjusting parameters and applying the setting (with an additional step for each area). With the Canvas, the user adjusts the parameters and apply the setting in one action. Most people valued this directness of the Canvas, as it cost less effort. The decreased effort lead to more finetuning of the light during the interaction: "The light changes immediately when moving [the token on the Canvas]. [With the Floorplan] you need to change the light $a$ bit and apply the setting again if you are not satisfied with [the light setting]. That is easier with [the Canvas]" (P11).

The two-dimensional field of light parameters that the Canvas shows (due to the chosen sequence of interaction) was used as a way to estimate the added value of starting an interaction. People estimated whether engaging with the interface was worth the effort, as they could assess how much the light could still be changed by looking at the position of the token in relation to the canvas. In this way, the overview formed a reminder to interact: "The [Canvas" printed preview] was my favorite because I could just glance up at it and see not only the current state as in the LED [preview of the Pointer], but the current state in relation to the possibilities. So, 'it's quite warm', for example. Or 'it could be warmer', or 'this is as warm as it can go'." (P6). Also, people could remember the position of the token for light settings they liked, thus forming sorts of presets in their memory. "I just knew: on the top right, is nice and bright." (P7). Theoretically, this was also possible with the other two interfaces by looking at the position of the parameter sliders but this was not mentioned by participants. Possibly because of the lower visibility of the Floorplan and Pointer.

\section{Representation of light}

Each interface uses a different medium to show a representation of the envisioned lighting adjustment, based on colour temperature and intensity). The Floorplan uses screen-based graphics, the Pointer uses an LED light, and the Canvas has printed graphics. Our findings show that a preview is a necessary form of feedback upon interaction, which increased the feeling of control. "Of course [a preview] is necessary, otherwise you won't know what you are doing." (P11). However, the preview was used to understand the functionality of a slider, rather than as a preview of the actual output of the lighting. "I did not use it as main reference of how the light was supposed to be. I think I usually just changed it and if I didn't like it I changed it again." (P16). This made accuracy of the representation less important, as long as adjustments could be made easily. "After using the thing twice you kind of know what to expect from it. [The preview] is not super important. It is necessary, but it is not a big deal if it deviates a bit [from the lighting]." (P11). 
In the Floorplan, the preview was often not noticed as such by participants, because it blended in with the other digital graphics. "Did the button change during interaction with [the Floorplan]? I didn't notice that. I guess a preview is useful but it's very cartoonish [in the Floorplan interface]. I think the LED preview is better." (P8). The light-based preview of the Pointer was generally liked because it is the same medium as the output system. However, the RGBLED did not accurately represent the resulting light, which was generally seen as a downside. Surprisingly, on a scale representing quality of the preview from 1 (low quality) to 5 (high quality), the digital print of the Canvas scored very high $(75 \%$ rated 3.9 or higher, means 4.3$)$, in spite of its low accuracy. This is partly because of the previously described overview that the mapping of light parameters gives: all possible colours can be previewed at once. This is also because of its abstraction: accuracy was not expected in the printed graphics, while it was expected in the lightbased representation. "I like [the printed preview] because it was an analogy. I know it is not giving me this red or this blue, but it is easier to learn how it would affect it." (P12).

Overall, none of the media (screen-based, light-based, or print) is generally better. It is important to have a representation as feedback on an action to increase the feeling of control, and to make the preview stand out from the rest of the interface. High accuracy is not always necessary as long as people's expectations of accuracy for the chosen medium are met.

\section{CONCLUDING REMARKS}

In this paper, we presented a long-term qualitative evaluation of three interfaces - the Floorplan, Pointer and Canvas - to investigate the influence of interface characteristics (ownership, distribution, and purpose, interaction modality, sequence of interaction and representation of light) on the user experience and use of a shared lighting system in a living lab office environment.

During the study, the living lab was occupied daily and each interface was used at least once every day. While the frequency of interaction differed per person, there were multiple interactions per day. The qualitative and quantitative results of the study show that participants were positive about all three interfaces. Moreover, we can conclude that the variations on interface characteristics were successfully represented in the three implementations, as participants were able to recognize and evaluate them.

The variations in the interface characteristics clearly affected people's experience and use of the lighting system. We found that the distribution, ownership and purpose affect how shared the interface and interaction feels to participants, and how much people include others in their lighting adjustments. Visibility of the interaction is a requirement for this sharedness. Distribution and purpose influence the effort that an interaction takes: having to walk to, or having to locate an app raises a threshold to interact. Moreover, a visible interface reminds people of the possibility to interact. The modality of the interaction can affect engagement with the system and the feeling of control over the light: the interfaces with more tangibility were scored higher on both factors. The sequence of interaction has to fit the context and common use patterns. Since people mainly adjusted the lamps in the area they were located themselves, an emphasis on the location of the adjustment (as the Floorplan and Pointer gave) was sometimes experienced as superfluous. Lastly, having a preview, or representation of the light, increases the feeling of being in control. However, this preview helps more with understanding the interface and light parameters than with estimating the impact of an adjustment on the lighting and the room. Surprisingly, the medium of that representation does not seem to matter much, as long as the accuracy lives up to the expectations people have of the chosen medium.

The identified effects of interface characteristics upon experience and use show that the characteristics represent important decisions when designing interfaces for a shared lighting system, and shared systems in general. What the most suitable alternative is for each characteristic depends on the context, controllable parameters, and intended use, and should therefore be considered carefully. The overview of the relations between characteristics and effects (figure 10) can fuel these considerations. However, one should be careful to use the identified relations as design guidelines, for three reasons. First, we combined design decisions on five characteristics in only three interfaces, which makes it difficult to clearly parse out the contribution of each design choice to the outcome. Second, the participants of the study were interaction design students and thus more knowledgeable on the subject than regular office workers. Third, the findings are based on one group of participants with their own group dynamics -that used three interfaces in a fixed order. So, based on our results, we identified relevant design considerations, and we present promising hypothesis on the relations between characteristic and effect that require further research.

There are several future steps we aim to take ourselves. To exclude effects of group dynamics and order, we plan to conduct the study again with a new group of participants and a different order of interfaces. Next to this, we aim to do a more extensive analysis (including all qualitative data) that focuses on the social aspects of shared lighting interaction. In this way, we expect to contribute to a better understanding of the relevance of design choices regarding interface characteristics for interaction with shared systems.

\section{ACKNOWLEDGEMENTS}

We thank our participants for their effort and openhearted accounts. This work was performed within the joint research program on Intelligent Lighting between TU/e and Koninklijke Philips N.V. and within the OpenAIS project, which has received funding from the European Union's Horizon 2020 research and innovation programme under grant agreement No 644332. 


\section{REFERENCES}

1. Dzmitry Aliakseyeu, Bernt Meerbeek, Jon Mason, Remco Magielse, and Susanne Seitinger. 2016. Peripheral Interaction with Light. In Peripheral Interaction, Challenges and Opportunities for HCI in the Periphery of Attention, Saskia Bakker, Doris Hausen and Ted Selker (eds.). Springer International Publishing, 207-235. http://doi.org/10.1007/978-3-319-29523-7

2. Apple. The smart home just got smarter. Retrieved March 31, 2017 from http://www.apple.com/lae/ios/home/

3. Saskia Bakker, Elise Van Den Hoven, and Berry Eggen. 2012. Acting by Hand: Informing Interaction Design for the Periphery of People's Attention. Interact. Comput. 24, 3, 119-130. http://doi.org/10.1016/j.intcom.2012.04.001

4. Saskia Bakker and Karin Niemantsverdriet. 2016. The Interaction-Attention Continuum: Considering Various Levels of Human Attention in Interaction Design. International Journal of Design 10, 2, 1-14.

5. Belkin. WeMo Home Automation. Retrieved March 31, 2016 from http://www.belkin.com/us/Products/ homeautomation/c/wemo-home-automation/

6. Peter R. Boyce, Neil H. Eklund, and S. Noel Simpson. 2000. Individual Lighting Control: Task Performance, Mood, and Illuminance. Journal of the Illuminating Engineering Society 29, 1, 131-142. http://doi.org/10.1080/00994480.2000.10748488

7. Peter R. Boyce, Jennifer A. Veitch, Guy R. Newsham, et al. 2006. Occupant use of switching and dimming controls in offices. Lighting Research and Technology 38, 4, 358376. http://doi.org/10.1177/1477153506070994

8. Virginia Braun and Victoria Clarke. 2006. Using thematic analysis in psychology. Qualitative Research in Psychology 3, 2, 77-101. http://doi.org/10.1191/1478088706qp063oa

9. Barry Brumitt and Jonathan J. Cadiz. 2001. "Let There Be Light" Examining Interfaces for Homes of the Future. Proceedings of IFIP INTERACT01: Human-Computer Interaction, 375-382.

10. Cisco. Cisco Digital Ceiling. Retrieved March 31, 2017 from http://www.cisco.com/c/en/us/solutions/ workforceexperience/digital-ceiling.html

11. Nils Dahlbäck, Arne Jönsson, and Lars Ahrenberg. 1993. Wizard of Oz studies - why and how. IUI '93 Proceedings of the 1st international conference on Intelligent user interfaces, 193-200. http://doi.org/10.1145/169891.169968

12. Tom Djajadiningrat, Stephan Wensveen, Joep Frens, and Kees Overbeeke. 2004. Tangible products: Redressing the balance between appearance and action. Personal and Ubiquitous Computing 8, 5, 294-309. http://doi.org/10.1007/s00779-004-0293-8

13. Elgato. Avea. Retrieved March 31, 2017 from https://www.elgato.com/en/smart/avea

14. Ulrich Engelke, Mariska G.M. Stokkermans, and Michael J. Murdoch. 2013. Visualizing lighting with images: converging between the predictive value of renderings and photographs. Proc. SPIE 8651, Human Vision and Electronic Imaging XVIII. http://doi.org/10.1117/12.2008465

15. Enlight consortium. Enlight: Energy efficient and intelligent lighting systems. Retrieved March 31, 2017 from http://www.enlight-project.eu

16. Thomas Erickson and Wendy A. Kellogg. 2000. Social Translucence: An Approach to Designing Systems That Support Social Processes. ACM Transactions on Computer-Human Interaction 7, 1, 59-83. http://doi.org/10.1145/344949.345004

17. Google. Get to know Google Home. Retrieved March 31, 2017 from https://madeby.google.com/home/

18. Valentin Heun. 2015. Reality Editor. Retrieved March 31, 2016 from http://www.valentinheun.com/portfolio/ reality-editor-smarter-objects/

19. Eva Hornecker and Jacob Buur. 2006. Getting a Grip on Tangible Interaction: A Framework on Physical Space and Social Interaction. CHI '06, ACM, 437-446. http://doi.org/10.1145/1124772.1124838

20. Elise van den Hoven, Evelien van de Garde-Perik, Serge Offermans, Koen van Boerdonk, and Kars-Michiel H. Lenssen. 2013. Moving tangible interaction systems to the next level. Computer 46, 8, 70-76. http://doi.org/10.1109/MC.2012.360

21. Elise van den Hoven and Ali Mazalek. 2011. Grasping gestures: Gesturing with physical artifacts. Artificial Intelligence for Engineering Design, Analysis and Manufacturing 25, 3, 255-271. http://doi.org/10.1017/S0890060411000072

22. Ikea. IKEA TRÅDFRI Connected lighting. Retrieved March 31, 2017 from http://www.ikea.com/se/sv/ catalog/categories/departments/living_room/36812/

23. Arjan de Kok. 2016. The new way of working: Bricks, bytes, and behavior. The Impact of ICT on Work, 9-40. http://doi.org/10.1007/978-981-287-612-6_2

24. Andrés Lucero, Tatiana Lashina, Elmo Diederiks, and Tuuli Mattelmäki. 2007. How probes inform and influence the design process. Proceedings of the 2007 conference on Designing pleasurable products and interfaces - DPPI '07, August, 377. http://doi.org/10.1145/1314161.1314195

25. Andrés Lucero, Jon Mason, Alexander Wiethoff, Bernt Meerbeek, Henrika Pihlajaniemi, and Dzmitry Aliakseyeu. 2016. Rethinking our interactions with light. Interactions 6, October, 54-59. http://doi.org/http://dx.doi.org/10.1145/3003334

26. Remco Magielse, Bart Hengeveld, and Joep Frens. 2013. Designing a light controller for a multi-user lighting environment. Proceedings of the 5th International Congress of International Association of Societies of Design Research (IASDR 2013).

27. Remco Magielse and Serge A. M. Offermans. 2013. Future lighting systems. CHI '13 Extended Abstracts on Human Factors in Computing Systems on - CHI EA '13, 2853. http://doi.org/10.1145/2468356.2479545 
28. Remco Magielse and Serge A. M. Offermans. 2013. Lithne - A Platform for Interaction Designers to Develop Interactive Networked Environments. Proceedings - 9th International Conference on Intelligent Environments, IE 2013, 137-144. http://doi.org/10.1109/IE.2013.14

29. Karin Niemantsverdriet, Mendel Broekhuijsen, Harm van Essen, and Berry Eggen. 2016. Designing for Multi-User Interaction in the Home Environment: Implementing Social Translucence. International Conference on Designing Interactive Systems (DIS'16), ACM, 13031314. http://doi.org/10.1145/2901790.2901808

30. Karin Niemantsverdriet, Harm van Essen, and Berry Eggen. 2017. A perspective on multi-user interaction design based on an understanding of domestic lighting conflicts. Personal and Ubiquitous Computing 21, 2, 371389. http://doi.org/10.1007/s00779-016-0998-5

31. Serge A.M. Offermans. 2016. Interacting with Light. Doctoral thesis. Eindhoven University of Technology, Eindhoven, The Netherlands.

32. Serge Offermans, Harm van Essen, and Berry Eggen. 2014. User Interaction with Everyday Lighting Systems. Personal and Ubiquitous Computing 18, 8, 2035-2055. http://doi.org/10.1007/s00779-014-0759-2

33. Osram. LIGHTIFY - smart connected light. Retrieved March 31, 2017 from https://innovation.osram.com/ connected-lighting-en/

34. Osram. LIGHTIFY Pro - intelligent, networked light for offices, lawyers' and doctors' practices and shops. Retrieved March 31, 2017 from

35. Philips. Hue. Retrieved March 31, 2016 from http://www2.meethue.com/en-XX/

36. Philips. Connected lighting for Offices. Retrieved March 31, 2017 from http://www.lighting.philips.co .uk/systems/system-areas/office-and-industry/ offices/connected-lighting.html

37. Philips. Hue Tap. Retrieved March 31, 2016 from http://www2.meethue.com/en-us/productdetail/philipshue-tap-switch

38. Seyed Amir Sadeghi, Panagiota Karava, Iason Konstantzos, and Athanasios Tzempelikos. 2016. Occupant interactions with shading and lighting systems using different control interfaces: A pilot field study. Building and Environment 97, 177-195. http://doi.org/10.1016/j.buildenv.2015.12.008

39. Bart Salters, Michael Murdoch, Dragan Sekulovksi, ShihHan Chen, and Pieter Seuntiens. 2012. An evaluation of different setups for simulating lighting characteristics. Proc. SPIE 8291, Human Vision and Electronic Imaging XVII, 1-13. http://doi.org/10.1117/12.912107
40. Tor Sørensen, Oskar D. Andersen, and Timothy Merritt. 2015. "Tangible Lights": In-Air Gestural Control of Home Lighting. Proceedings of the 9th International Conference on Tangible, Embedded, and Embodied Interaction - TEI '15 Work-in-Progress. http://doi.org/10.1145/2677199.2687909

41. Brigg Ullmer and Hiroshi Ishii. 2000. Emerging frameworks for tangible user interfaces. IBM Systems Journal 39, 3\&4, 915-931.

42. Jennifer A Veitch. 2011. Workplace design contributions to mental health and well-being. HealthcarePapers 11 Spec No, 38-46. Retrieved January 16, 2017 from http://www.ncbi.nlm.nih.gov/pubmed/24917255

43. Jennifer A. Veitch, Guy R. Newsham, P. Boyce, and C. Jones. 2008. Lighting appraisal, well-being and performance in open-plan offices: A linked mechanisms approach. Lighting Research and Technology 40, 2, 133151. http://doi.org/10.1177/1477153507086279

44. Thomas C. F. Van de Werff, Harm A. Van Essen, and Berry Eggen. 2017. The Impact of the Internet of Lighting on the Office Lighting Value Network. Journal of Industrial Information Integration, accepted for publication. http://doi.org/10.1016/j.jii.2017.03.002

45. Thomas van de Werff, Karin Niemantsverdriet, Harm van Essen, and Berry Eggen. 2016. Designing Multi-user Lighting Interfaces : Four strategies to implement Social Translucence. DIS '16 Companion Proceedings, 137-140.

46. Alison Williams, Atkinson Barbara, Karina Garbesi, and Page Erik. 2012. Lighting Controls in Commercial Buildings. LEUKOS: The Journal of Illuminating Engineering Society of North America 8, 3, 161-180. http://doi.org/10.1582/LEUKOS.2012.08.03.001

47. John Zimmerman, Jodi Forlizzi, and Shelley Evenson. 2007. Research through design as a method for interaction design research in HCI. Proceedings of the SIGCHI conference on Human factors in computing systems - CHI '07, 493-502. http://doi.org/10.1145/1240624.1240704

48. Amazon Echo. Retrieved March 31, 2017 from https:/www.amazon.com/Amazon-Echo-BluetoothSpeaker-with-WiFi-Alexa/dp/B00X4WHP5E

49. Processing.org. Retrieved March 31, 2017 from https://processing.org/

50. Qualitative Data Analysis Software MAXQDA for Windows \& Mac - MAXQDA. Retrieved March 31, 2017 from http://www.maxqda.com/ 